\section{Implants that stay where you put them}

Trycare Ltd is the UK Distributor for Adin Dental Implant Systems, the world's tenth largest manufacturer of dental implants. Clinical tests in universities and hundreds of thousands of successful restorations make Adin implants first choice for many implantologists.

Adin's spiral design implants are suitable for all indications and exceptional for immediate loading. Their surfaces have been treated for faster osseointegration and New York University research indicates that they integrate as well as Nobel, Straumann and Astra.

Their unique thread and tip slices through bone, compressing and condensing on the way, offering outstanding primary stability and optimal restorative orientation. Whilst their surface treatment has been shown to increase osseointegration. Macroscopic grooves on the surface of the implant thread double the surface area of most other implants and give better bone-to-implant contact.

Adin spiral design implants can be redirected during placement with little or no reduction of stability, the dual cutting blade edge helping to minimise the osteotomy site and trauma to bone and surrounding soft tissue. This makes them ideal for thinner bone ridges.

There will be an opportunity to learn more about Adin implants at the Trycare September 2020 Extravaganza, London, 11 September. The speakers are world renown Patrick Palacci, Ulf Nannmark, Dr Paul Schuh and Marcos White from the UK. The event will deliver six hours of enhanced CPD for just $£ 395.00$ plus vat.

For further information visit the Trycare website, www.trycare. co.uk/adin, or request one of their Adin Catalogues. To request a catalogue contact your local representative or call 01274885544.

\section{Opt-on loupes and lights}

Appointed UK distributor for the complete range of Orange Dental Opt-on loupes and lights, Trycare believe that purchasing loupes doesn't need to be a hassle.

Buying loupes from other companies can be a long-winded procedure involving at least one long surgery visit, lots of timeconsuming measuring and a long delay waiting for the loupes. And, if there is a problem, the loupes have to be returned to the manufacturer for correction. Similarly, if components become worn or the operator's prescription changes they have to be returned for repair or alteration, taking weeks and great expense.

Opt-on loupes and lights have a stylish and robust modular design which means they can be easily modified or repaired without

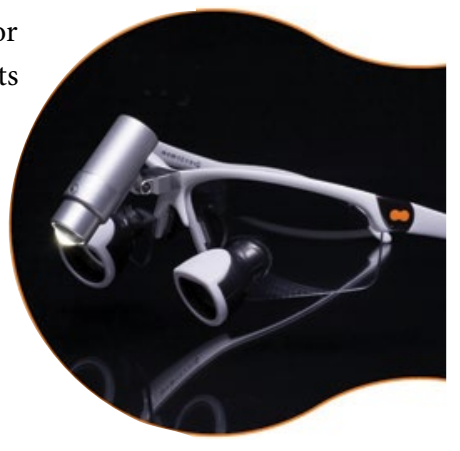
returning them to the manufacturer. If the operator wears glasses and their prescription changes, it's easy to do. If the operator wants to switch from glasses to lenses they can, simply by putting in or taking out a prescription adaptor. Even changing the appearance is quick and easy too.

Incredibly light, just $32 \mathrm{~g}$, Opt-on loupes are comfortable to wear all day long, without the need to take them off. Available in a choice of $2.7 \times$ full arch and $3.3 \times$ three-quarter arch variants, in a wide range of colours, they have adjustable arms for enhanced comfort and are supplied in six working distances from $25 \mathrm{~cm}$ to $50 \mathrm{~cm}$.

To complement Opt-on loupes Trycare offer a choice of wired and cordfree Spot-on lights.

For further information visit the Trycare website, www.trycare.co.uk/ orangedental, contact your local representative or call 01274885544.

\title{
Spot the very early signs of caries
}

The CALCIVIS imaging system demonstrates the dental team's commitment to preventive dental care. Using bioluminescence to visualise active demineralisation on the surfaces of the teeth,

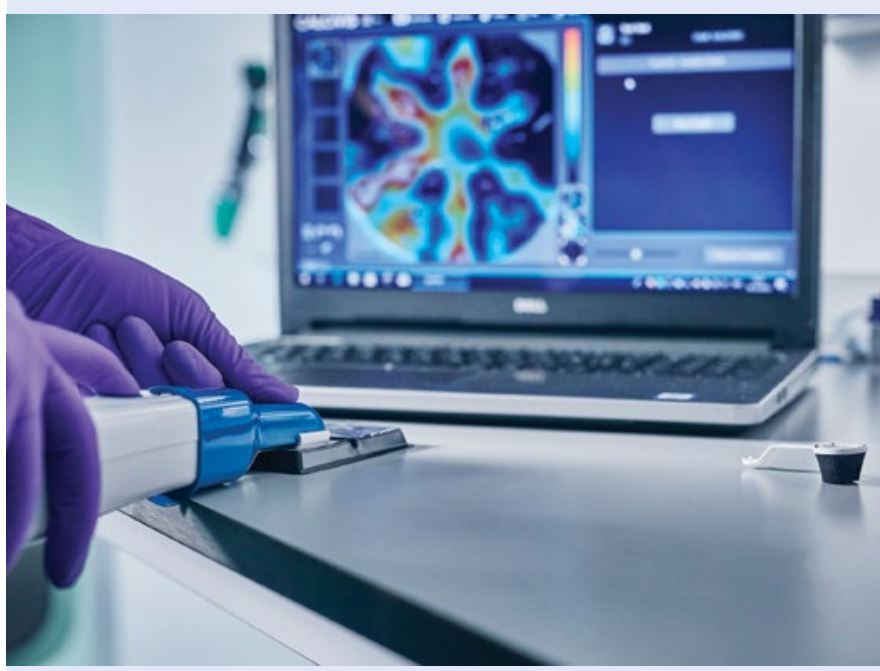

this device enables practitioners to see the very early signs of the caries disease process in time to initiate first response, reparative therapy. The evidence-based images also allow patients to better understand their oral health, which motivates them to comply with preventive measures to avoid further damage to the teeth. Revolutionary technology such as this keeps existing patients interested and attracts new patients to the practice. Furthermore, it has the potential to generate additional revenue to facilitate further investment and progression.

As further scientific and technological developments are made, patients' expectations of the dental profession will continue to rise. Consequently, practices need to build on these advancements and evolve with technology to add value to both the practice and the patient experience. Innovation provides enhanced diagnostics, better disease management and modern, progressive dental care that can help practices to meet their patients' demands and maintain a competitive edge.

For more information visit www.calcivis.com, call 01316585152 oremailinfo@calcivis.com. 